\title{
Relatively Evaluation of PAPR Reduction in OFDM Gadget the Use of Iterative Partial Transmit Sequence Set of Rules below Distinctive Modulation Schemes
}

\author{
Himanshi Shrivastava, Prof. Ashish Verma
}

\begin{abstract}
The OFDM is one of the hooked up multicarrier modulation strategies, which gives excessive spectral performance, low implementation complexity, much less vulnerability to echoes and non-linear distortion. apart from the above blessings currently this approach is utilized by nearly all wi-fi requirements and above. the first-rate important shortcoming inside the implementation of this gadget is the excessive PAPR (peak-to-average power ratio) of this device. There are various methods to overcome this drawback. Partial Transmit sequence (PTS) is one of them. in this paper, a modified model of the PTS has been proposed i.e. Iterative PTS (I-PTS), which reduces the overall performance gap of the conventional PTS method underneath exceptional modulation schemes. The overall performance of device is simulated through using MATLAB software software.
\end{abstract}

Keywords: Orthogonal frequency division multiplexing (OFDM), Peak-to-average Power ratio (PAPR), Bit Error Rate (BER), and Complementary Cumulative Distribution Function(CCDF), Partial Transmit sequence (PTS),Iterative Partial Transmit sequence (I-PTS) etc.

\section{Introduction}

Orthogonal Frequency division Multiplexing (OFDM) has various advantages such as better spectral typical overall performance, tons less sensitivity to sample timing offsets, robustness with appreciate to multi-direction fading, excessive immunity to inter-photo interference and capability to control up with frequency selective fading of wideband communication with tolerable complexity. the ones elements makes OFDM healthful for incorporating in several WI-FI technology like virtual Video Broadcasting(DVB-T), WI-FI LAN, long term Evolution (LTE) and so forth[1]. OFDM has numerous poor additives too .It has noise like amplitude, with a very large dynamic variety and it's miles more touchy to company frequency offset and go with the flow in comparison to unmarried provider systems, because of that, it suffers from the trouble of high Peak to average power ratio (PAPR), which reduces the performance of high power Amplifier (HPA) at transmitter give up. PAPR forces the HPA to feature beyond its maximum applicable linear variety, which finally ends up nonlinear distortion in the transmitted signal. PAPR may be decreased through numerous strategies like Coding, Interleaving, and Selective Mapping (SLM), Partial Transmit series (PTS), Tone Reservation and Tone Injection and so on. [2].PAPR discount techniques may be labeled into classes:

1) Which reduces PAPR in advance than modulation.

2) That reduces PAPR after modulation.

This paper analyzes the complexity component in Partial Transmit series (PTS) and gives an green set of rules i.e. Iterative-PTS (I-PTS), which reduces the performance gap OF the traditional PTS method the usage of unique modulation strategies .in segment II, fundamentals of Orthogonal Frequency division Multiplexing (OFDM) has been mentioned. segment III offers with basics of Partial Transmit series method. segment IV discusses unique and
Iterative Flipping algorithm the usage of specific modulation techniques. In segment $\mathrm{V}$, a simulation result indicates the overall performance of proposed approach, and a conclusion has been discussed in section VI.

\section{Fundamentals of OFDM Signal}

Orthogonal Frequency division Multiplexing (OFDM)" has been brought, (OFDM) is multi-service modulation (MCM) Scheme approach used for 4th technology (4G) wireless verbal exchange wherein a supply signal might be cut up into numerous narrowband channels at extraordinary frequencies such as the block of $\mathrm{N}$ records symbols $\mathrm{Xn}$, $\mathrm{n}=$ zero, $1 \ldots \ldots \ldots, \mathrm{N}-1$ that paperwork the one OFDM signal, is transmitted .figure(1) suggests the block diagram of OFDM signal wherein the bit flow is split in to various orthogonal sub-companies, each of which modulated at low rate [3] . each OFDM signals modulates a exclusive subprovider from set $\mathrm{fn}, \mathrm{n}=0,1, \ldots \ldots \mathrm{N}-1$. The $\mathrm{N}$ sub-service are orthogonal to every other, i.e, $\mathrm{fn}=\mathrm{n} \Delta \mathrm{f}$. where $\Delta \mathrm{f}=1 / \mathrm{NT}$ and $\mathrm{T}$ = image duration, multicarrier signal such as $\mathrm{N}$ sub-service is as follows:

$$
\mathrm{Xn}=\frac{1}{\sqrt{N}} \sum_{\mathrm{n}=0}^{\mathrm{N}-1} \mathrm{Xne}^{\mathrm{j} 2 \pi \mathrm{tfn}}
$$

in which, $0 \leq t \leq T$ because of the multi carrier modulation scheme OFDM will reduce The loss of data on the receiver cease. as a result, it reduces the retransmission charge, which in consequences low strength consumption, less time and cheaper in fee.

PAPR of transmitted sign is given as:

$$
\begin{array}{r}
\text { PAPR }=\frac{\text { PEAK POWER }}{\text { AVERAGE POWER }} \\
\text { PAPR }=\frac{\max [|x(n)| 2]}{E[|x \boldsymbol{n}| 2]}
\end{array}
$$

\section{Volume 6 Issue 1, January 2017




\section{International Journal of Science and Research (IJSR) \\ ISSN (Online): 2319-7064}

Index Copernicus Value (2015): 78.96 | Impact Factor (2015): 6.391

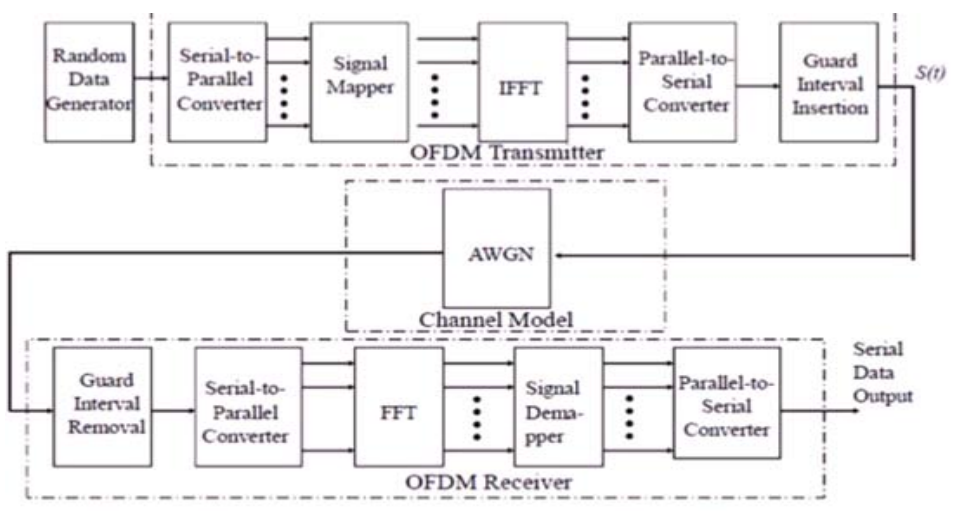

Figure 1: Block Diagram of OFDM system

\section{Complementary Cumulative Distribution function (CCDF)}

CCDF calculates the possibility that the PAPR of a records block exceeds a given threshold PAPR0 and be computed thru Monte Carlo Simulation [4].

So as to extract useful records from this noise-like signal, we want a statistical description of the energy tiers on this sign, and a CCDF curve gives just that. A CCDF curve suggests how a whole lot time the signal spends at or above a given strength degree. The power level is expressed in $\mathrm{dB}$ relative to the common electricity.

From the principal limit theorem, for big variety of values of $\mathrm{N}$, the actual and imaginary values of $\mathrm{x}(\mathrm{t})$ will become Gaussian allotted. The amplitude of the OFDM signal, therefore, has a Rayleigh distribution with zero suggest and a variance of $\mathrm{N}$ times the variance of 1 complex sinusoid. The Complementary Cumulative Distribution characteristic
(CCDF) of the PAPR of $\mathrm{N}$ symbols of a information block with Nyquist sampling fee is described as

\section{$\mathbf{P}_{\mathrm{r}}\left(\mathrm{PAPR} \geq \mathbf{P A P R}_{\mathbf{0}}\right)=1-\mathbf{P}_{\mathrm{r}}\left(\mathbf{P A P R} \leq \mathrm{PAPR}_{\mathbf{0}}\right)$}

$$
=1-\left(1-e^{-P A P R}\right)^{N}
$$

\section{Partial Transmit Sequence}

In partial transmit sequence. The whole facts block is partitioned $\mathrm{X}$ into $\mathrm{M}$ disjoint sub-blocks as $\mathrm{X}=\left[\mathrm{X}_{0}, \mathrm{X}_{1, \mathrm{XN}-1}\right]^{\mathrm{T}}$ and then calculating individual IFFT' $S$ as

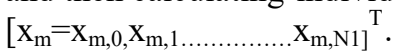

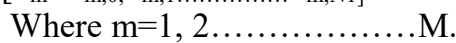

THEN, quite a few these disjoint devices are multiplied with random section vector, this is given in Block diagram in figure 2 .

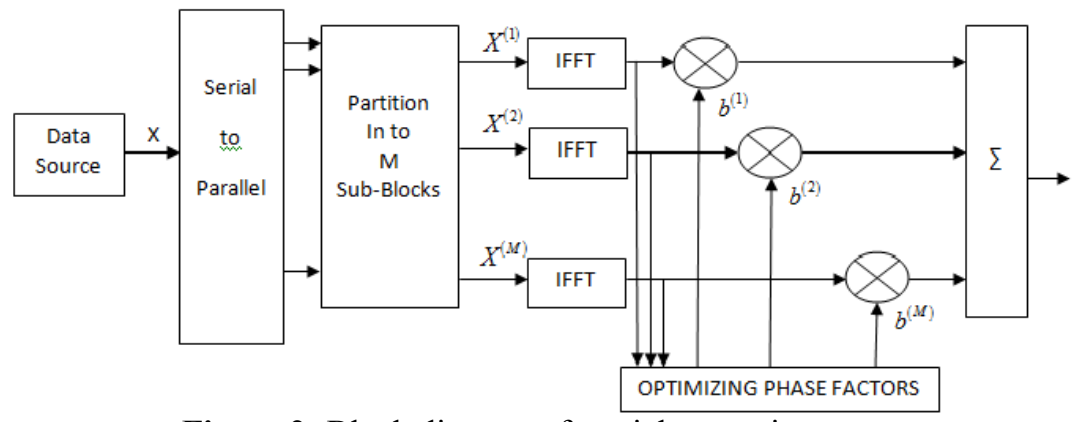

Figure 2: Block diagram of partial transmit sequence

The segment rotation vectors $\mathrm{b} 1, \mathrm{~b} 2, \ldots, \mathrm{bM}$ are multiplied with the above generated disjoint subsets. This is the level, in which section optimization strategies are hired to lower the PAPR of the sign. After this, simplest the units which have maximum efficient section vectors with lowest PAPR are transmitted.

PTS approach includes desire of OFDM sign with lesser PAPR sets, consequences extra complicated in nature. So, the transmitter desires to track the information of the dispatched information blocks There are three styles of subblock partitioning scheme: adjacent, interleaved and pseudorandom partitioning. The enter sign (candidate sign) is the sum of the made of segment rotation vector and corresponding sub-block. For this reason, the candidate sign is given through

$$
\mathrm{X}^{\mathrm{c}}=\sum_{m=1}^{M} \text { bm Xm }
$$

Where $\mathrm{c}=1,2 \ldots \ldots \ldots \ldots \ldots . \mathrm{c}$

\section{Drawback of PTS}

the primary trouble with PTS method is its complexity because it requires an exhaustive seek over all the combos of allowed segment factors [5], and because of this, the complexity will boom exponentially with respective the range of sub-blocks. Sub-block partitioning moreover influences the PAPR reduction typical performance in PTS. The PTS method works with an arbitrary sort of subproviders and any modulation scheme 


\section{International Journal of Science and Research (IJSR) \\ ISSN (Online): 2319-7064}

Index Copernicus Value (2015): 78.96 | Impact Factor (2015): 6.391

\section{Iterative Flipping PTS Technique}

Cimini and Sollenberger's iterative flipping method is advanced as a sub-choicest method for the PTS set of rules. Of their original paper (10), they most effective use binary weighting factors. That is $\mathrm{bm}=1 \mathrm{or} \mathrm{bm}=-1$. These may be extended to more segment elements. The set of rulesis as follows.

1) After dividing the facts block into $M$ disjoint subblocks, one assumes that $\mathrm{bm}=1,(\mathrm{~m}=1,2, \ldots \ldots, \mathrm{M})$ for all of subblocks and calculates PAPR of the OFDM signal.

2) Then one changes the sign of the first subblock section issue from 1 to $-1(\mathrm{~b} 1=-1)$, and calculates the PAPR of the signal once more.

3) If the PAPR of the formerly calculated sign is greater than that of the present day signal, hold $\mathrm{b} 1=-1$. Otherwise, revert to the preceding segment component, $\mathrm{b} 1=1$.

4) Think one chooses $b 1=-1$. Then the primary section aspect is decided, and thus saved constant for the remaining a part of the algorithm. Next, we comply with the identical method for the second subblock. when you consider that one assumed all of the phase elements have been 1 , within the $2 \mathrm{~d}$ subblock, one additionally changes $\mathrm{b} 2=1$ to $\mathrm{b} 2=-1$, and calculates the PAPR of the OFDM signal.

If the PAPR of the formerly calculated sign is bigger than that of the present day sign, preserve $\mathrm{b} 2=-1$. Otherwise, revert to the preceding segment component, $\mathrm{b} 2=1$. This indicates the manner with the second subblock is similar to that with the first subblock. One keeps performing this process iteratively till one reaches the stop of subblocks (Mth subblock and segment component bM). A similar method turned into additionally proposed via Jayalath and Tellambura (nine). The difference among the Jayalath and Tellambura's technique and that of Cimini and Sollenberger is that, inside the former, the flipping manner does no longer necessarily go to the end of subblocks (Mth block). To reduce computational complexity, the flipping is stopped earlier than the quit of the complete method if the desired PAPR OFDM signal performed at that factor.
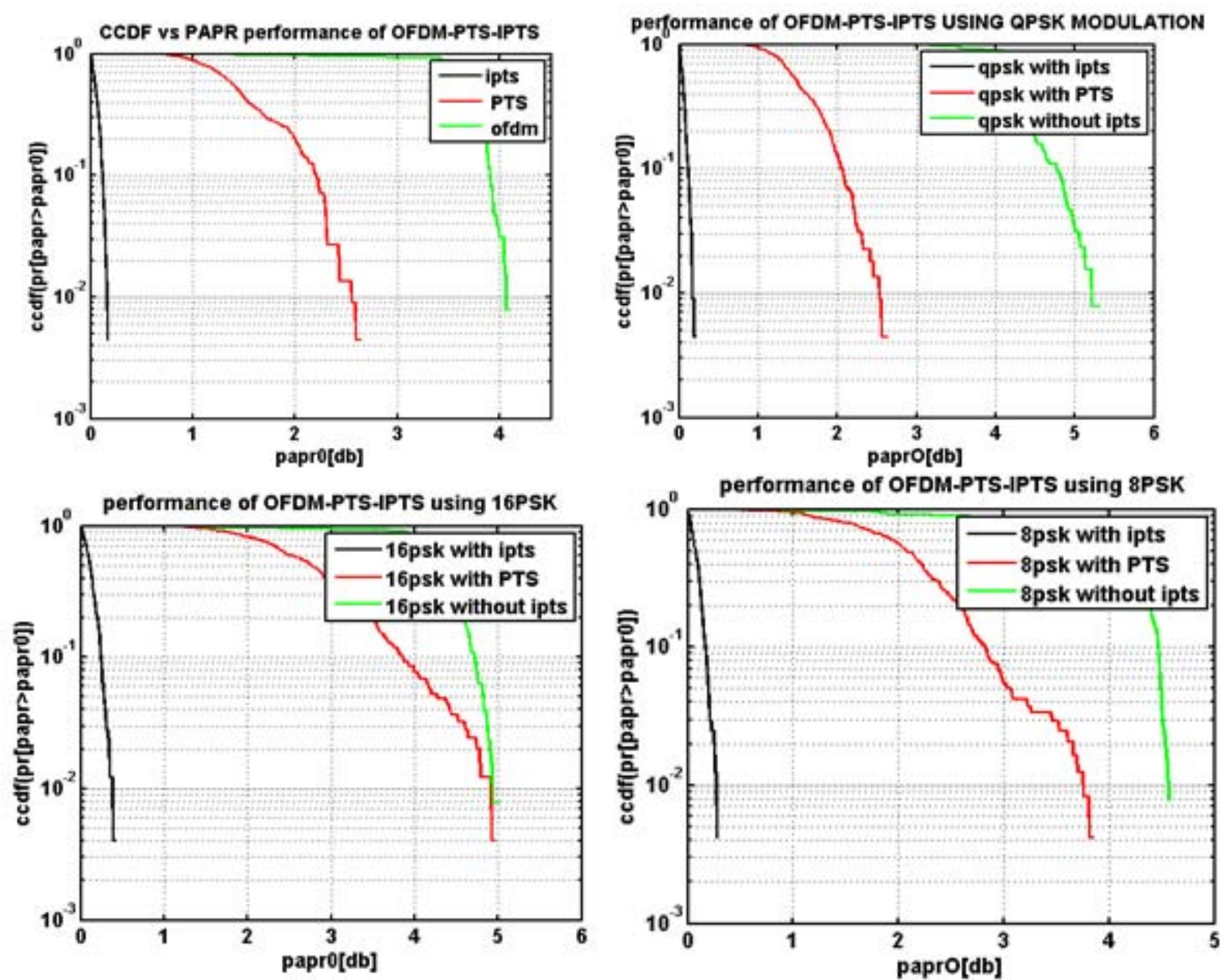
International Journal of Science and Research (IJSR)

ISSN (Online): 2319-7064

Index Copernicus Value (2015): 78.96 | Impact Factor (2015): 6.391

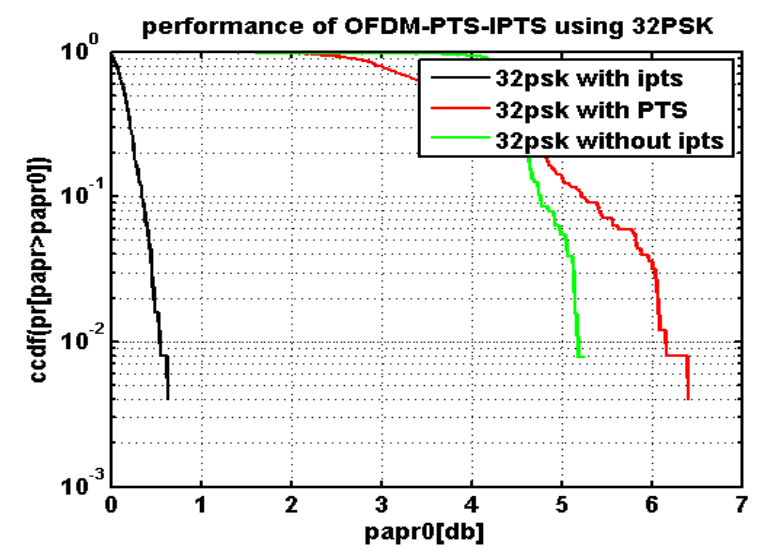

Table I: Simulation Parameters

\begin{tabular}{|c|c|}
\hline Simulation Parameters & Type/Value \\
\hline Number of sub-carriers (N) & 128 \\
\hline Number of sub-blocks (M) & $32,16,8,4$ \\
\hline Cyclic Prefix & $(0.1 *$ number of sub-block $)$ \\
\hline Oversampling Factor (L) & $4,8,16,32$ \\
\hline Constellation Size & QPSK,8PSK,16PSK,32PSK \\
\hline OFDM Blocks & 10,000 \\
\hline
\end{tabular}

Table 2: Comparison of OFDM, PTS and I-PTS Technique under Different Modulation Schemes

\begin{tabular}{|c|c|c|c|}
\hline \multicolumn{4}{|c|}{ PAPR $(\mathrm{db})$} \\
\hline Modulation & OFDM Signal & PTS Signal & I-PTS Signal \\
\hline QPSK & 7.6242 & 4.5267 & 3.1982 \\
\hline 8PSK & 7.3125 & 4.1702 & 3.7773 \\
\hline 16PSK & 8.4556 & 5.4150 & 4.4624 \\
\hline 32PSK & 7.6369 & 4.8268 & 4.6738 \\
\hline
\end{tabular}

\section{Conclusion}

In this paper, a new set of rules I-flipping has been proposed for PAPR reduction in OFDM system the usage of specific modulation techniques. To similarly examine their PAPR reduction overall performance for various sub-carriers, we examine the I-flipping and pts with the authentic ofdm scheme. We also evaluate the OFDM sign ,pts signal and that i-pts sign the use of distinctive MODULATION techniques(QPSK,8PSK,16PSK and 32 PSK) From the simulation end result it may be seen that proposed I-Flipping scheme provide foremost PAPR discount with decreased complexity at every modulation strategies. in addition studies paintings can be prolonged to reduce the PAPR in addition to computational complexity with versions in subblocks, iterations, samples and so forth. green PAPR reduction scheme is one that reduces the PAPR to minimal without affecting a great deal to the performance and additionally with low implementation fee and must be key to future wireless high velocity verbal exchange structures.

\section{References}

[1] M. Singh and S. K. Patra, "Analysis of PAPR Reduction Schemes in LTE-OFDM System," in IEEE International Conference on Advanced Research in Engineering and Technology (ICARET 2013, India), pp. 568-571, 2013.

[2] S. H. Han and J. H. Lee, "An overview of peak-toaverage power ratio reduction techniques for multicarrier

transmission,"

IEEE

WirelessCommunications, no. April, pp. 56-65, 2005.

[3] Ramjee Prasad, OFDM for Wireless Communications Systems. Artech House Universal Personal Communications Library, 2004.

[4] S. Muller and J. Huber, "OFDM with reduced peak-toaverage power ratio by optimum combination of partial transmit sequences," Electronics Letters, vol. 33, no. 5, pp. 368-369, 1997.

[5] C. Tellambura, "Improved phase factor computation for the PAR reduction of an OFDM signal using PTS," IEEE Communications Letters, vol. 5, pp. 135-137, Apr. 2001.

[6] Y. S. Cho, J. Kim, W. Y. Yang, and Chung G. Kang, MIMO-OFDM wireless communications with MATLAB. John Wiley \& Sons, Inc., 2010.

[7] L. Cimini and N. Sollenberger, "Peak-to-average power ratio reduction of an OFDM signal using partial transmit sequences," IEEE Communications Letters, vol. 4, pp. 86-88, Mar. 2000.

[8] G. Zhu and T. Jiang, "Extended iterative flipping algorithm for PAPR reduction in OFDM systems," in 2008 IEEE Third International Conference on Communications and Networking in China, pp. 10181022, 2008.

[9] A. D. S. Jayalath and C. Tellambura "Adaptive PTS approach for reduction of peak-toaverage power ratio of OFDM signal," Electronics Letters, pp. 1226-1228, vol. 36 , no. 14 6th July 2000

[10] L. J. Cimini, Jr. and N.R. Sollenberger, "Peak-toaverage power ratio reduction of an OFDM signal using partial transmit sequences," IEEE Communication Letters, vol. 4, pp. 86-88, Mar. 2000 